\title{
Virulence Plasmid (pYV)-Associated Expression of Phenotypic Virulent Determinants in Pathogenic Yersinia Species: A Convenient Method for Monitoring the Presence of pYV under Culture Conditions and Its Application for Isolation/Detection of Yersinia pestis in Food
}

\author{
Saumya Bhaduri and James L. Smith \\ Molecular Characterization of Foodborne Pathogens Research Unit, Eastern Regional Research Center, Agricultural Research Service, \\ U.S. Department of Agriculture, 600 East Mermaid Lane, Wyndmoor, PA 19038, USA
}

Correspondence should be addressed to Saumya Bhaduri, saumya.bhaduri@ars.usda.gov

Received 30 May 2011; Revised 23 June 2011; Accepted 27 June 2011

Academic Editor: Didier Montet

Copyright ( $) 2011$ S. Bhaduri and J. L. Smith. This is an open access article distributed under the Creative Commons Attribution License, which permits unrestricted use, distribution, and reproduction in any medium, provided the original work is properly cited.

\begin{abstract}
In Yersinia pestis, Y. pseudotuberculosis, and Y. enterocolitica, phenotypic expression of virulence plasmid (pYV: 70-kb)-associated genetic determinants may include low-calcium response (Lcr, pinpoint colony, size $=0.36 \mathrm{~mm})$, colony morphology $($ size $=$ $1.13 \mathrm{~mm}$ ), crystal violet (CV) binding (dark-violet colony), Congo Red (CR) uptake (red pinpoint colony, size $=0.36 \mathrm{~mm}$ ), autoagglutination $(\mathrm{AA}=$ cells agglutinate), and hydrophobicity ( $\mathrm{HP}=$ clumping of cells). Y. pseudotuberculosis is chromosomally closely related to $Y$. pestis; whereas, Y. enterocolitica is chromosomally more distantly related to Y. pestis and Y. pseudotuberculosis. All three species demonstrate Lcr, CV binding, and CR uptake. The colony morphology/size, AA, and HP characteristics are expressed in both Y. pseudotuberculosis and Y. enterocolitica but not in $Y$. pestis. Congo red uptake in $Y$. pestis was demonstrated only on calcium-deficient CR magnesium oxalate tryptic soy agar (CR-MOX), whereas this phenotype was expressed on both CR-MOX and low-calcium agarose media in Y. pseudotuberculosis and Y. enterocolitica. These phenotypes were detectable at $37^{\circ} \mathrm{C}$ within $24 \mathrm{~h}$ in Y. enterocolitica and Y. pseudotuberculosis but did not appear until $48 \mathrm{~h}$ in $Y$. pestis due to its slower growth rate at $37^{\circ} \mathrm{C}$. The pYV is unstable (i.e., easily lost under a variety of culture conditions) in all three species but is more unstable in Y. pestis. The specific CR uptake by Y. pestis in CR-MOX and the delayed time interval to express Lcr and CR uptake provide a means to differentiate $Y$. pestis from Y. enterocolitica and Y. pseudotuberculosis. These differences in pYV expression in Y. pestis can be used for its isolation and detection in food.
\end{abstract}

\section{Introduction}

The genus Yersinia consists of 11 species, but only Y. pestis, Y. enterocolitica, and Y. pseudotuberculosis are pathogenic to humans. Yersinia pestis is considered to be ancestrally related to Y. pseudotuberculosis; however, Y. pseudotuberculosis behaves phenotypically and clinically like Y. enterocolitica [1]. The three species are quite diverse in the diseases they cause; $Y$. enterocolitica and $Y$. pseudotuberculosis induce gastroenteritis when consumed in contaminated food and have been isolated from patients with diarrhea. Yersinia pestis is the agent of bubonic plague and can cause oropharyngeal plague as a result of the consumption of inadequately cooked goat and camel meat or handling of meat from infected animals [2-5]. The risk, morbidity, and mortality of contracting plague through the consumption of food deliberately contaminated with $Y$. pestis are currently unknown but potentially real. Furthermore, the identification of multidrug-resistant strains [6] and the potential use of this pathogen for the deliberate contamination of food could cause plague in large populations. 


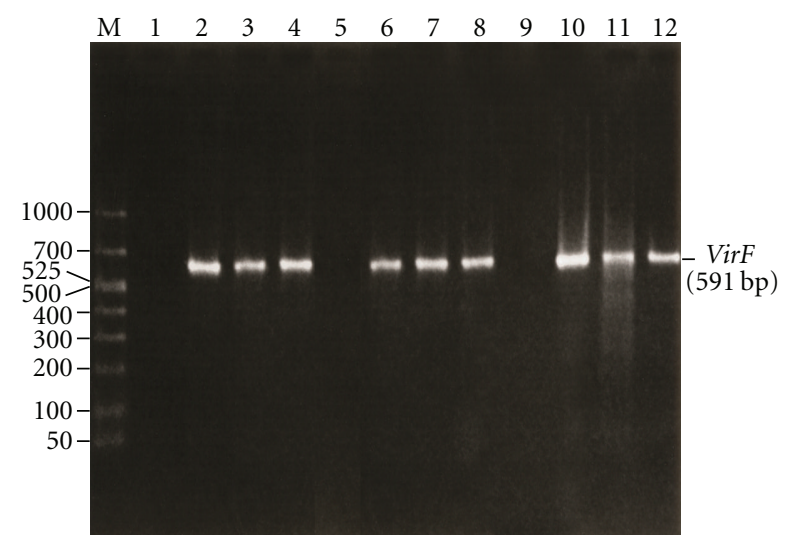

FIGURE 1: Confirmation of the presence of pYV in the original strains, cells in red pinpoint colonies, and cells in the white border around a red pinpoint colony from CR-MOX by PCR assay targeting a key regulatory gene $v i r F$, which encodes a transcriptional activator for the expression of pYV-encoded outer membrane protein Yop51. The primer pairs (5'-TCATGGCAGAACAGCAGTCAG- $3^{\prime}$ and $5^{\prime}$ ACTCATCTTACCATTAAGAAG- $3^{\prime}$ ) for detection of the $\operatorname{vir} F$ gene (430- to 1020-nucleotide region) amplified a 591 base pair (bp) product from the virulence plasmid. Lane M, 50-1,000 bp ladder marker; lanes, 1, 5, and 9 showing the absence of 591-bp product in cells of the white borders of $Y$. enterocolitica, Y. pseudotuberculosis, and $Y$. pestis, respectively; lanes 2, 6, and 10 showing the presence of 591-bp product in the original strains of $Y$. enterocolitica, $Y$. pseudotuberculosis, and $Y$. pestis respectively before phenotypic evaluation; lanes 3, 7, and 11 showing the presence of 591-bp product in cells of the red pinpoint colonies of $Y$. enterocolitica, $Y$. pseudotuberculosis, and $Y$. pestis, respectively, and lanes 4, 8, and 12 showing the presence of 591-bp product within cells of red pinpoint colonies surrounded by white border of $Y$. enterocolitica, $Y$. pseudotuberculosis, and Y. pestis respectively [7].

Three plasmids are involved in the virulence of $Y$. pestis: (a) pYV (virulence plasmid, 70-kb, Yops, type III secretion system), (b) pFra/pMT1 (96.2-kb, murine toxin: phospholipase, F1 capsule-like antigen), and (c) pCP1/pPst/pPla (9.6$\mathrm{kb}$, plasminogen activator) $[8,9]$. Among these plasmids, the pYV-encoded type III secretion system (Yops) promotes cytotoxicity and the common symptoms of plague [8]. The pYV of all three species are of the same size and genetically highly conserved $[8,10-12]$. It encodes the ability to target lymph tissues during infection and has genetic determinants essential for infection and overcoming host defense mechanisms $[8,10-12]$. In the three species, carriage of pYV is responsible for the calcium-dependent growth phenotype at $37^{\circ} \mathrm{C}$. The cultivation of pYV-bearing cells in lowcalcium/calcium-deficient media elicits a $\mathrm{Mg}^{2+}$-dependent low-calcium response (Lcr), which results in the production of pYV-encoded virulence-associated antigens ( $\mathrm{V}$ and $\mathrm{W}$ ), and a series of released proteins (Yops). The low-calcium response is expressed phenotypically on solid media by the formation of pinpoint colonies [8, 10-12]. Furthermore, $\mathrm{pYV}$ in $Y$. enterocolitica has been correlated with several other in vitro characteristics, which are phenotypically expressed at $37^{\circ} \mathrm{C}$. The well-characterized pYV-associated virulence determinants include colony morphology/size,
Lcr, crystal violet (CV) binding, Congo red (CR) uptake, autoagglutination (AA), hydrophobicity (HP), mannoseresistant haemagglutination, expression of surface fibrillae, and serum resistance [11-13]. However, the expression of these physiological traits at $37^{\circ} \mathrm{C}$ also fosters the loss of pYV and the concomitant disappearance of the associated phenotypes. Since Y. pestis and Y. pseudotuberculosis have nearly identical chromosomal DNA sequences and are distantly related to pathogenic $Y$. enterocolitica $[1,12,14]$, the purpose of this paper is to review whether the phenotypic characteristics induced by pYV are expressed in $Y$. pestis and $Y$. pseudotuberculosis and to determine the growth conditions required for the expression of these phenotypic characteristics. In addition, the detection and isolation of $Y$. pestis by monitoring the presence of pYV-encoded Lcr and CR-uptake virulence phenotypes are discussed.

\section{Expression of pYV-Associated Phenotypic Virulence Determinants}

In Y. pestis, Y. pseudotuberculosis, and Y. enterocolitica, the expression of phenotypic virulence characteristics is encoded by pYV [7]. A derivative of a clinical Y. pestis (KIM5: Kurdistan Iran man) strain lacking the chromosomal 102$\mathrm{kb}$ Pgm locus (pigmentation), but harboring all three virulence plasmids (pYV, pFra/pMT1, and F1) $[7,9]$, was used for our study. The Pgm locus is only present in $Y$. pestis. This strain is conditionally virulent (a conditional mutant is only infectious if inoculated intravenously) and can be used in a BL2 laboratory facility [7, 9]. This strain shows CR-uptake in $Y$. pestis due to the presence of pYV; whereas, another derivative of a clinical strain of $Y$. pestis, the Kuma strain, contains the chromosomally encoded determinant, $\mathrm{Pgm}^{+}$for CR-uptake but lacks pYV [7]. Clinical isolates of $Y$. enterocolitica (serotype O:3; strain GER) and Y. pseudotuberculosis (serotype O:1b; strain $\mathrm{PB} 1 /+$ ) were also used in our study $[7,13]$. The presence of pYV in $Y$. pestis, Y. enterocolitica, and Y. pseudotuberculosis was confirmed by a PCR assay targeting a key regulatory gene, virF, present on pYV (Figure 1, lanes 2, 6, and 10) [15]. The primers (5'-TCATGGCAGAACAGCAGTCAG- $3^{\prime}$ and $5^{\prime}$-ACTCATCTTACCATTAAGAAG-3') for the detection of the $\operatorname{virF}$ gene (430- to 1020-nucleotide region) amplified a 591-base pair (bp) sequence from the virulence plasmid [15]. Yersinia pestis Kuma strain did not show the presence of pYV by the PCR assay.

In our study, the pYV-negative derivatives $\left(\mathrm{P}^{-}\right)$of $Y$. pestis KIM5, Y. pseudotuberculosis, and Y. enterocolitica were obtained from large flat colonies, which emerged spontaneously from pYV-positive $\left(\mathrm{P}^{+}\right)$cultures growing at $37^{\circ} \mathrm{C}$ on brain heart infusion agarose with $238 \mu \mathrm{M}$ $\mathrm{Ca}^{2+}$ (BHO) [16] and were used as negative controls. The expression of pYV-encoded genetic determinants in Y. pestis, Y. pseudotuberculosis, and Y. enterocolitica was evaluated [7]. When $\mathrm{P}^{+}$and $\mathrm{P}^{-}$strains were cultivated at $37^{\circ} \mathrm{C}$ for $24-$ $48 \mathrm{~h}$ on a low-calcium brain heart infusion agarose with $238 \mu \mathrm{M} \mathrm{Ca}^{2+}$ (BHO), low-calcium tryptic soy broth agarose with $311 \mu \mathrm{M} \mathrm{Ca}^{2+}$ (TSO), and calcium-deficient magnesium 


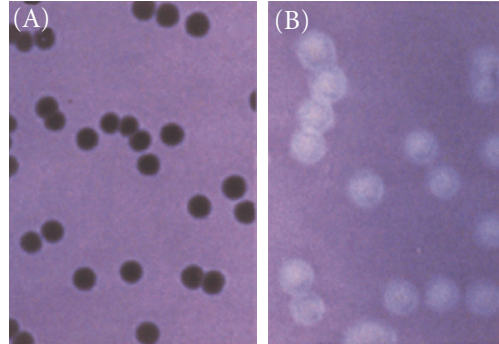

(A) CV binding of virulent $\mathrm{P}^{+}$strain showing small dark-violet colonies (B) Avirulent $\mathrm{P}^{-}$strain showing large white colonies

(a) Crystal violet Binding

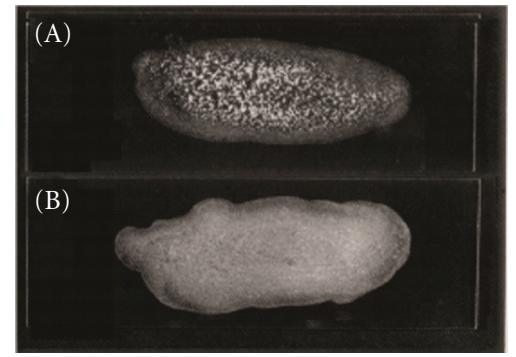

(A) Virulent $\mathrm{P}^{+}$strain agglutinated forming clumps

(B) Avirulent $\mathrm{P}^{-}$strain remained dispersed

(c) Hydropholicity test

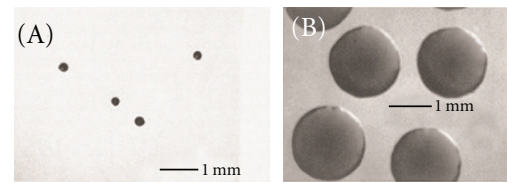

(A) Virulent $\mathrm{P}^{+}$strain appeared as pinpoint colonies $(0.36 \mathrm{~mm}$ in diameter)

(B) Avirulent $\mathrm{P}^{-}$strain showing large colonies (1.37 $\mathrm{mm}$ in diameter)

(e) Low calcium response

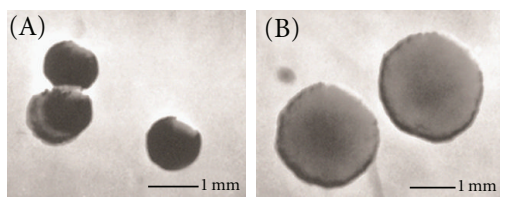

(A) Virulent $\mathrm{P}^{+}$strain appeared as small colonies (1.13 $\mathrm{mm}$ in original diameter)

(B) Avirulent $\mathrm{P}^{-}$strain showing large colonies (2.4 $\mathrm{mm}$ in diameter)

(b) Colony morphology/size

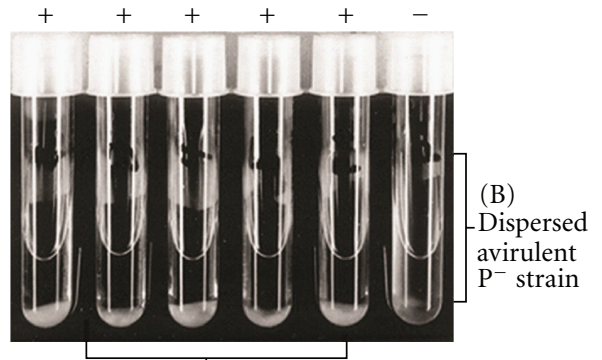

(A) Agglutinated virulent $\mathrm{P}^{+}$strain

(d) Autoagglutination test
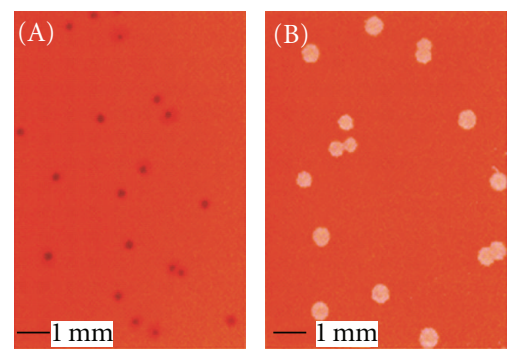

(A) Virulent $\mathrm{P}^{+}$strain appeared as red pinpoint colonies $(0.36 \mathrm{~mm}$ in diameter)

(B) Avirulent $\mathrm{P}^{-}$strain showing large white colonies ( $1.37 \mathrm{~mm}$ in diameter)

(f) Congo red binding uptake

Figure 2: Evaluation of pYV-associated virulent phenotypes of pathogenic Yersinia species [7].

oxalate agar with tryptic soy agar (TSA) with $20 \%$ Dgalactose, $0.25 \mathrm{M}$ sodium oxalate, and $0.25 \mathrm{M}$ magnesium chloride (MOX), the $\mathrm{P}^{+}$cells of $Y$. enterocolitica and $Y$. pseudotuberculosis produced pinpoint colonies $(0.36 \mathrm{~mm}$ in diameter; Figure $2(\mathrm{e})(\mathrm{A})$ at $24 \mathrm{~h}$, whereas $Y$. pestis $\mathrm{P}^{+}$ formed pinpoint colonies at $48 \mathrm{~h}$. The $\mathrm{P}^{-}$cells from each representative strain formed much larger colonies $(1.37 \mathrm{~mm}$ in diameter; Figure 2(e)(B). The size and colony morphology of each $\mathrm{P}^{+}$strain when grown on $75 \mu \mathrm{g} / \mathrm{mL}$ Congo red (CR) containing $\mathrm{BHO}$ (CR-BHO), TSO (CR-TSO), and 1\% CR containing (CR-MOX) showed identical expression of Lcr as well as CR-uptake $(0.36 \mathrm{~mm}$ diameter; Figure $2(\mathrm{f})(\mathrm{A})$ under all these conditions (Table 1). CR-uptake was demonstrated as bright red pinpoint colonies in $Y$. enterocolitica and $Y$. pseudotuberculosis on all three media (Table 2). However, CR-uptake of $Y$. pestis gave a less intense red color as compared to that of Y. enterocolitica and Y.pseudotuberculosis on CR-BHO and CR-TSO (Table 2). An increase of CR concentration in BHO and TSO to $100 \mu \mathrm{g} / \mathrm{mL}, 150 \mu \mathrm{g} / \mathrm{mL}$, and $200 \mu \mathrm{g} / \mathrm{mL}$ did not increase the color intensity of $Y$. pestis $\mathrm{P}^{+}$colonies as compared to colonies of $Y$. enterocolitica and Y. pseudotuberculosis. On the basis of color contrast between the bacterial colony and the medium, CR-MOX was more suitable to show CR-uptake in $Y$. pestis as compared to CR-BHO (Table 2). The $\mathrm{P}^{-}$cells from each representative strain failed to bind CR and formed much larger colonies 


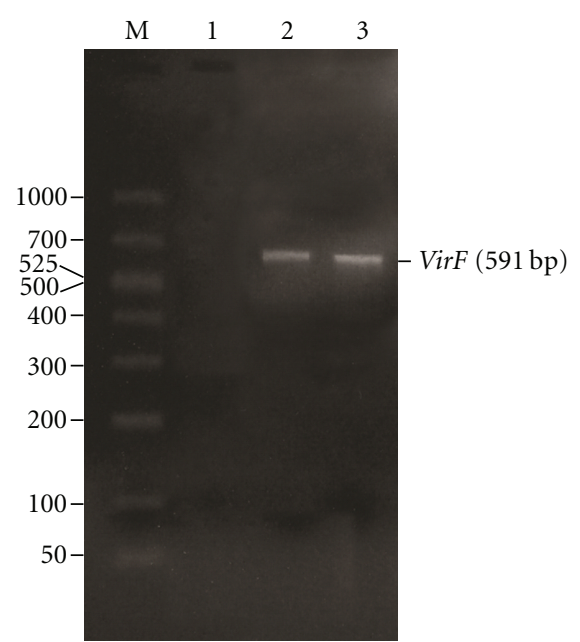

Figure 3: Detection of pYV in cells recovered from red pinpoint colony and subcultured in brain heart infusion broth at $28^{\circ} \mathrm{C}$ by PCR assay targeting $\operatorname{virF}$ gene of pYV. The primer pairs ( $5^{\prime}$-TCATGGCAGAACAGCAGTCAG- $3^{\prime}$ and $5^{\prime}$-ACTCATCTTACCATTAAGAAG-3') for detection of the virF gene (430to 1020-nucleotide region) amplified a 591 base-pair (bp) product from the virulence plasmid. Lane $M, 50-1,000$ bp ladder marker; lane 1 showing the absence of 591-bp product in $Y$. pestis; lanes 2 and 3 showing the presence of 591-bp product in $Y$. enterocolitica and $Y$. pseudotuberculosis, respectively.

(1.37 $\mathrm{mm}$ in diameter; Figure 2(f)(B). The difference of CRuptake and the difference in timing of the expression of Lcr and CR-uptake in Y. pestis facilitate differentiating this species from $Y$. pseudotuberculosis and Y. enterocolitica. On calcium-adequate $(1500 \mu \mathrm{M})$ CR-BHA (brain heart infusion agar) and CR-TSA (tryptic soy agar), colonies of both $\mathrm{P}^{+}$and $\mathrm{P}^{-}$strains of $Y$. pseudotuberculosis and $Y$. pestis remained white or light orange similar to that reported for $Y$. enterocolitica $(14,17)$. The calcium concentration in CR-BHO $\left(238 \mu \mathrm{M} \mathrm{Ca}^{2+}\right)$ and CR-TSO $\left(311 \mu \mathrm{M} \mathrm{Ca}^{2+}\right)$ is relatively low, whereas, in CR-MOX, sodium oxalate is used to sequester calcium leading to a calcium-deficient medium. Thus, the CR-uptake in Y.pestis is more dependent on calcium depletion than that of $Y$. enterocolitica and $Y$. pseudotuberculosis. Moreover, Y. pestis Kuma, $\left(\mathrm{Pgm}^{+}, \mathrm{pYV}^{-}\right)$ failed to bind CR on CR-MOX and formed large white or light orange colonies (1.37 $\mathrm{mm}$ in diameter) [7].

That the expression of CR-uptake on CR-MOX is specifically encoded by pYV was further confirmed using a number of derivatives of clinical strains of $Y$. pestis (CDC A1122, CO99.3015, Yokohama, P12, D1, D3, D5, D7, D9, D13, D17) containing Pgm but lacking the pYV [7, 10]. These $\mathrm{Pgm}^{+} / \mathrm{pYV}^{-}$strains did not bind CR on CR-MOX. These observations indicate that the CR-uptake in $Y$. pestis grown on CR-MOX is associated with pYV. Thus, pYV-encoded CRuptake is independent of $\mathrm{Pgm}^{+}$and that the Pgm locus is not expressed on CR-MOX at $37^{\circ} \mathrm{C}$. The $\mathrm{CR}$ phenotype is encoded by pYV only on calcium-depleted medium. Thus, CR-uptake in $Y$. pestis grown on CR-MOX is independent of chromosomally encoded CR binding virulence determinants $\left(\mathrm{Pgm}^{+}\right)$and is associated with the presence of $\mathrm{pYV}$.
Another characteristic feature of the CR-uptake in $\mathrm{P}^{+}$ strains of $Y$. enterocolitica is the appearance of a white opaque circumference around the red center after $48 \mathrm{~h}$ of incubation at $37^{\circ} \mathrm{C}$ [17]. This characteristic colony type was also observed in Y.pseudotuberculosis after $48 \mathrm{~h}$ of incubation and in $Y$. pestis after $72 \mathrm{~h}$ of incubation. The timing of this colonial characteristic is another parameter that can be used for the identification of $\mathrm{P}^{+}$strains of $Y$. pseudotuberculosis and $Y$. pestis $[7,17]$. The cells in red pinpoint colonies (Figure 1, lanes 3, 7, and 11) and red centered colonies surrounded by a white border (Figure 1, lanes 4, 8, and 12) contained pYV in Y. pseudotuberculosis and $Y$. pestis similar to the cells reported in $Y$. enterocolitica [7, 17]. Cells in the surrounding white border (Figure 1, lanes 1, 5, and 9) do not contain pYV as demonstrated by PCR. When the pYV-bearing cells recovered from red pinpoint colonies were subcultured in BHI broth (brain infusion broth) at $28^{\circ} \mathrm{C}$ for $18 \mathrm{~h}, Y$. enterocolitica and Y. pseudotuberculosis showed the presence of pYV by PCR (Figure 3, lanes 2 and 3 ) and pYV-associated phenotypic characteristics, while $Y$. pestis did not harbor pYV (Figure 3, lane 1) under the same conditions (Table 1). This showed that pYV is more stable in Y. enterocolitica and Y. pseudotuberculosis than in Y.pestis. Thus, CR uptake can also be used to isolate viable $\mathrm{P}^{+}$cells in $Y$. pseudotuberculosis and Y. enterocolitica [7, 17-19].

The flooding of colonies of $\mathrm{P}^{+}$strains on BHA, TSA, CR$\mathrm{BHO}, \mathrm{CR}-\mathrm{TSO}$, and CR-MOX grown at $37^{\circ} \mathrm{C}$ with $\mathrm{CV}$ solution at a concentration of $100 \mu \mathrm{g} / \mathrm{mL}$ showed that $\mathrm{P}^{+}$cells from all three Yersinia species bound $\mathrm{CV}$ and produced darkviolet colonies (Table 1; Figure $2(\mathrm{a})(\mathrm{A})$ ). The $\mathrm{P}^{-}$colonies did not bind $\mathrm{CV}$ and remained white (Figure $2(\mathrm{a})(\mathrm{B})$ ). The $\mathrm{CV}$ - and CR-binding assays can effectively identify individual pYV-bearing colonies from a mixed culture of $\mathrm{P}^{+}$and $\mathrm{P}^{-}$ strains $[7,17,20]$. The CR uptake is unrelated to CV binding; these two phenomena are independent since CV uptake is not related to Lcr.

The colony size of $\mathrm{P}^{+}$cells in $Y$. enterocolitica and $Y$. pseudotuberculosis was smaller $(1.13 \mathrm{~mm}$ in diameter; Figure 2(b)(A) than corresponding $\mathrm{P}^{-}$cells when grown on $\mathrm{BHA}$ and TSA at $37^{\circ} \mathrm{C}(2.4 \mathrm{~mm}$ in diameter: Figure $2(\mathrm{a})(\mathrm{B})$ [16], whereas, $\mathrm{P}^{+}$and $\mathrm{P}^{-}$cells of $Y$. pestis were approximately the same size $(1.3-1.4 \mathrm{~mm} \mathrm{SD} \pm 0.11$ in diameter $)$ at $37^{\circ} \mathrm{C}$. This may be due to the fact that the optimum growth temperature of $Y$. pestis is $28^{\circ} \mathrm{C}(7,9,11,14)$. Hydrophobicity by latex particle agglutination was positive (Figure $2(\mathrm{c})(\mathrm{A})$ ) for pYV-bearing $Y$. enterocolitica and $Y$. pseudotuberculosis but negative for $\mathrm{P}^{-}$cells (Figure $2(\mathrm{c})(\mathrm{B})$ ). Y. pestis showed no $\mathrm{HP}$ when pregrown cells were tested from CR-BHO, CRTSO, CR-MOX, BHA, and TSA (Table 1). Thus, HP of $Y$. enterocolitica and $Y$. pseudotuberculosis was expressed in low calcium, calcium-deficient, and calcium-adequate media, indicating that HP is also a non-Lcr property.

The autoagglutination test in Eagle minimal medium supplemented with $10 \%$ fetal bovine serum was positive (Figure 2(d)(A)) for pYV-bearing $Y$. enterocolitica and $Y$. pseudotuberculosis but not for $\mathrm{P}^{-}$cells (Figure $2(\mathrm{~d})(\mathrm{B})$ ). Yersinia pestis cultures failed to autoagglutinate (Table 1). In both the HP and AA tests, $\mathrm{P}^{-}$strains were negative for the three species. The explanation for the absence of expression 
TABle 1: Comparison of selected phenotypic expression of pYV-bearing Y. enterocolitica, Y.pseudotuberculosis, and Y. pestis (adapted from [7]).

\begin{tabular}{|c|c|c|c|c|c|c|c|c|}
\hline Organism $^{\mathrm{a}}$ & Strain & $\mathrm{CM}^{\mathrm{b}}$ & CV binding ${ }^{\mathrm{c}}$ & $\mathrm{Lcr}^{\mathrm{d}}$ & CR-uptake ${ }^{\mathrm{e}}$ & $\mathrm{AA}^{\mathrm{f}}$ & $\mathrm{HPg}^{\mathrm{g}}$ & Plasmid $^{\mathrm{h}}$ \\
\hline Y. enterocolitica & GER & + & + & + & + & + & + & + \\
\hline Y. enterocolitica-RE & GER & + & + & + & + & + & + & + \\
\hline Y.enterocolitica-C & GER & - & - & - & - & - & - & - \\
\hline Y.pseudotuberculosis & $\mathrm{PB} 1 /+$ & + & + & + & + & + & + & + \\
\hline Y.pseudotuberculosis-RE & $\mathrm{PB} 1 /+$ & + & + & + & + & + & + & + \\
\hline Y. pseudotuberculosis-C & $\mathrm{PB} 1 /+$ & - & - & - & - & - & - & - \\
\hline$Y$. pestis & KIM5 & - & + & + & + & - & - & + \\
\hline Y. pestis-RE & KIM5 & - & - & - & - & - & - & - \\
\hline$Y \cdot$ pestis-C & KIM5 & - & - & - & - & - & - & - \\
\hline pYV-less Y. pestis & Kuma & - & - & - & - & - & - & - \\
\hline
\end{tabular}

${ }^{a}$ Cells recovered from red pinpoint colonies and subcultured in $\mathrm{BHI}$ broth at $28^{\circ} \mathrm{C}$ are designated as RE. The pYV-negative strains of $Y$. enterocolitica, $Y$. pseudotuberculosis, and $Y$. pestis are designated as C (cured).

${ }^{\mathrm{b}} \mathrm{CM}$ : colony morphology. On calcium-adequate BHA $\left(1500 \mu \mathrm{M} \mathrm{Ca}^{2+}\right)$, and TSA $\left(1400 \mu \mathrm{M} \mathrm{Ca}^{2+}\right)$ the $\mathrm{P}^{+}$cells appeared as small colonies $(1.13 \mathrm{~mm}$ in diameter $)$ as compared to larger $\mathrm{P}^{-}$colonies $(2.4 \mathrm{~mm}$ in diameter).

${ }^{c} \mathrm{CV}$ binding: crystal violet binding. The $\mathrm{P}^{+}$cells appeared as small dark-violet colonies, and the $\mathrm{P}^{-}$cells showed large white colonies on calcium-adequate BHA $\left(1500 \mu \mathrm{M} \mathrm{Ca}^{2+}\right)$ and TSA $\left(1400 \mu \mathrm{M} \mathrm{Ca}^{2+}\right)$, low-calcium CR-BHO $\left(238 \mu \mathrm{M} \mathrm{Ca}^{2+}\right)$, CR-TSO $\left(311 \mu \mathrm{M} \mathrm{Ca}^{2+}\right)$, and calcium-deficient CR-MOX.

${ }^{\mathrm{d}}$ Lcr: low calcium response/calcium-dependent growth. $\mathrm{P}^{+}$cells appeared as pinpoint colonies (0.36 in diameter), and $\mathrm{P}^{-}$cells appeared large colonies (1.37 in diameter) on low-calcium CR-BHO $\left(238 \mu \mathrm{M} \mathrm{Ca}^{2+}\right)$, CR-TSO $\left(311 \mu \mathrm{M} \mathrm{Ca}^{2+}\right)$, and calcium-deficient CR-MOX.

${ }^{e} \mathrm{CR}-$ Uptake: Congo red-uptake. The $\mathrm{P}^{+}$cells appeared as red pinpoint colonies ( 0.36 in diameter), and the $\mathrm{P}^{-}$cells appeared large white or light orange colonies (1.13 mm in diameter) on calcium-deficient CR-MOX.

${ }^{\mathrm{f}} \mathrm{AA}$ : autoagglutination. The $\mathrm{P}^{+}$cells agglutinated. The $\mathrm{P}^{-}$cells remained dispersed.

gHP: hydrophobicity by latex particles. The $\mathrm{P}^{+}$cells formed clumps showing hydrophobicity. The $\mathrm{P}^{-}$cells remained dispersed.

hPlasmid: presence of 70-kb pYV by PCR assay.

TABLE 2: Effect of media on CR-uptake in pYV-bearing Y. enterocolitica, Y.pseudotuberculosis, and Y. pestis (adapted from [7]).

\begin{tabular}{lcccc}
\hline Organism $^{\text {a }}$ & Strain & CR-BHO & CR-TSO \\
\hline Y. enterocolitica & GER & + & + & + \\
Y. enterocolitica-RE & GER & + & - & + \\
Y. enterocolitica-C & GER & + & + \\
Y.pseudotuberculosis & PB1/+ & + & + \\
Y.pseudotuberculosis-RE & PB1/+ & - & - \\
Y.pseudotuberculosis-C & PB1/+ & - & - & + \\
Y. pestis & KIM5 & - & - \\
Y.pestis-RE & KIM5 & - & - \\
Y.pestis-C & KIM5 & - & - \\
pYV-less Y. pestis & Kuma & - & - \\
\hline
\end{tabular}

${ }^{a}$ Cells recovered from red pinpoint colonies and subcultured in BHI broth at $28^{\circ} \mathrm{C}$ are designated as RE. The pYV-negative strains of $Y$. enterocolitica Y. pseudotuberculosis, and Y. pestis are designated as C (cured).

Low-calcium: CR-BHO $\left(238 \mu \mathrm{M} \mathrm{Ca}^{2+}\right)$ and CR-TSO $\left(311 \mu \mathrm{M} \mathrm{Ca}^{2+}\right)$. CR-MOX (calcium deficient).

of the HP and AA phenotypic characteristics under the conditions described above in $Y$. pestis may be due to the lack of synthesis of pYV-associated surface factors essential for HP and AA or due to a structural/regulatory variability of pYV [21].

In conclusion, of the six pYV-associated phenotypes evaluated, only three phenotypes (Lcr, CR-uptake, and CV binding) were expressed in $Y$. pestis, while all six properties were expressed in Y. enterocolitica and Y. pseudotuberculosis. This differential expression of pYV-encoded phenotypes may be attributed to in vitro assay conditions although pYV is genetically highly conserved in all these species $[6,12,14,21]$. Thus, the pYV-encoded phenotypes can be used as virulence markers for these pathogens $[7,10,11,13]$. Although the chromosomal DNA sequence showed that $Y$. pestis and $Y$. pseudotuberculosis are nearly identical and closely related [1, 14], the latter exhibits the same expression of pYV-associated phenotypes as the more distantly related Y. enterocolitica and shows similar characteristics and clinical symptoms [1].

\section{Procedure to Monitor the Presence of pYV in Y. pestis Cells during Storage and Culturing by Using the Lcr-CR-Uptake Techniques}

The well-characterized pYV-associated virulence determinants can be used to determine plasmid maintenance, for isolation/detection, and as an indication of virulence for various serotypes of pYV-bearing $Y$. enterocolitica in food 
$[11,17-20,22-25]$, as well as to determine the presence of pYV in Y. pestis and Y. pseudotuberculosis [7]. The pYV is unstable in all three pathogens, and the loss of pYV after cultivation or during food processing results in avirulent clones (not lethal to mice; do not cause plague) [7, 11, 13, 26-30]. Repeated transfer of cultures, extended storage at $4^{\circ} \mathrm{C}$ or $-20^{\circ} \mathrm{C}$, and laboratory manipulation, as well as subculturing of $Y$. pestis at temperatures $>30^{\circ} \mathrm{C}$ leads to the loss of pYV $[7,29,30]$. Moreover, pYV is more unstable in $Y$. pestis (Figure 3, lane 1) than in of $Y$. enterocolitica and Y. pseudotuberculosis (Figure 3, lanes 2 and 3) [7, 29, 30]. The loss of pYV leads to the eventual overgrowth by cells lacking pYV and results in the loss of virulence and the concomitant disappearance of the $\mathrm{pYV}$-associated virulence characteristics $[7,13,25,27,28]$.

In a study on the growth of $Y$. pestis in ground beef, it was found that the cultures lost pYV during preparation of the inoculum [29]. It was not possible to maintain pYV in cells from the stock cultures using the standard procedures developed previously [13]. Thus, it was difficult to perform a study with $Y$. pestis, which reflected the actual behavior of pYV-bearing $Y$. pestis. In ground beef, the growth rates of pYV less cells were 0.096 and $0.287 \mathrm{CFU} / \mathrm{h}$ at 10 and $25^{\circ} \mathrm{C}$, respectively; [30] whereas, for pYV-bearing cells, the growth rates were $0.057 \mathrm{CFU} / \mathrm{h}$ and $0.233 \mathrm{CFU} / \mathrm{h}$ at 10 and $25^{\circ} \mathrm{C}$, respectively $[29,30]$. The difference in growth rate between pYV-positive and pYV-negative strains of $Y$. pestis was more pronounced at lower temperatures. There was no growth of the pYV-bearing strain at 0 and $4^{\circ} \mathrm{C}$ as compared to the growth rates of pYV-negative strains of 0.003 and $0.016 \mathrm{CFU} / \mathrm{h}$ at 0 and $4^{\circ} \mathrm{C}$, respectively, in ground beef [29]. Therefore, the lack of pYV leads to a faster growth rate and does not represent the true growth rate of the pYVbearing strain. Hence, it is very important to maintain $\mathrm{pYV}$ in $Y$. pestis to properly study the growth behavior of a pYV-bearing strain in order to develop a growth model for this pathogen in food. The unstable nature of pYV in $Y$. pestis necessitates an examination for the presence of pYV and its virulence characteristics throughout laboratory manipulation and investigations.

Bhaduri et al. [30] developed a procedure to monitor the presence of pYV in $Y$. pestis cells during storage and culturing by using the Lcr-CR-binding techniques [7, 30], PCR assays, and the expression of pYV-associated virulence characteristics. It is essential to confirm the presence of pYV in the experimental culture by demonstrating that virulence-associated phenotypes were present and to confirm the presence of the pYV-encoded virF gene by a PCR assay (Figure 4, lane 3) [7, 15, 30]. The procedures for monitoring the presence of $\mathrm{pYV}$ and differentiating $\mathrm{pYV}$ positive clones from $\mathrm{pYV}$-negative colonies during laboratory investigations are outlined in Table 3 [30]. As described in Table 3, the first step is to culture $Y$. pestis on CR-MOX and CR$\mathrm{BHO}$ to isolate pYV-bearing clones from the frozen stock culture. The pYV-positive colonies appeared as red pinpoint colonies $(0.36 \mathrm{~mm}$ in diameter) showing both Lcr and CRuptake whereas pYV-negative colonies appeared as much larger white or orange colonies $(1.37 \mathrm{~mm}$ in diameter) [ 7 , $17,30]$. Colony morphology and CR-uptake were used to

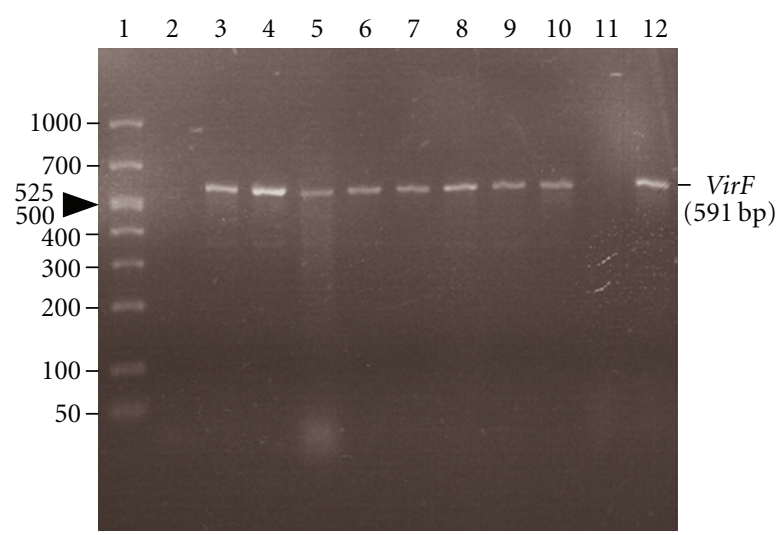

FIgURE 4: Confirmation of presence of pYV of $Y$. pestis in the original strain before subculturing, and CR-positive clones from CR-MOX, CR-BHO, and BHI broth using PCR assay targeting a key regulatory gene virF from $\mathrm{pYV}$. The primer pairs (5'-TCATGGCAGAACAGCAGTCAG-3' and $5^{\prime}$ ACTCATCTTACCATTAAGAAG- $3^{\prime}$ ) for detection of the $\operatorname{virF}$ gene (430- to 1020-nucleotide region) amplified a 591-bp-product from the virulence plasmid. The Lcr-CR ${ }^{+}$clones showed the presence of $591 \mathrm{bp}$ products from $\mathrm{pYV}$ (lanes $2-10$ and 12). Lane 1, 50-1,000 bp ladder marker; lane 2, negative control with no template; lane 3, original KIM5 strain as positive control; lanes 4, 5, Lcr-CR ${ }^{+}$colonies from the CR-MOX and CR-BHO respectively (Figure 5 ; no. 1); lane $6 \mathrm{BHI}$ broth (Figure 5; 1st passage; no. 2); lane 7 stock culture on CR-MOX (no. 3; 1st passage); lane 8 stock culture on CR-BHO (no. 3; 1st passage); lane $9 \mathrm{BHI}$ broth (no. 4, 2nd passage from CRMOX); lane BHI broth 10 (no. 4, 2nd passage from CR-BHO); lane 11 (no. 5, 2nd passage on CR-MOX) showing the absence of 591-bp product, and lane 12 (no. 5, 2nd passage on CR-BHO) [30].

differentiate between pYV-positive clones and pYV-negative colonies. The Lcr and CR positive clones were further confirmed as pYV positive by the PCR assay (Figure 4, lane 4: CR-MOX and lane 5: CR-BHO) and by pYV-associated Lcr, CR uptake, and CV-binding phenotypes (see Table 3). These pYV-bearing clones were inoculated into BHI broth for the preparation of frozen and working stock cultures as described in Table 3. Before frozen storage and preparation of working stock cultures, the culture prepared in BHI broth at $28^{\circ} \mathrm{C}$ was tested for the presence of pYV and its virulence-associated phenotypes (Figure 4, lane 6). The Lcr-CR-positive clones on CR-MOX were used as working stock cultures and could be used for 15 days for laboratory studies. After that period of storage, the red pinpoint colonies of $Y$. pestis lost pYV (Figure 4, lane 11). The CR-BHO medium was also successfully used to ensure the selection of PYV in $Y$. pestis although CR-uptake was not as intense as on CR-MOX. The Lcr-CR positive clones were used as working stock cultures from CR-BHO and could be stored for 30 days at $2^{\circ} \mathrm{C}$. To ensure the validity of this procedure for selecting $\mathrm{pYV}$ in $Y$. pestis cells, we also examined and monitored pYV stability during the subculturing of pYVbearing cells in BHI broth, CR-MOX, and CR-BHO. Yersinia pestis from stock cultures stored at $2^{\circ} \mathrm{C}$ on CR-MOX and CR-BHO were subcultured as explained in Figure 5 [30]. The presence of $\mathrm{pYV}$ in $Y$. pestis cells in each medium and 
TABLE 3: Isolation and maintenance of pYV in Y. pestis [30].

Day 1

(i) Frozen stock cultures were streaked onto CR-MOX and CR-BHO.

(ii) Plates were incubated at $37^{\circ} \mathrm{C}$ for $48 \mathrm{~h}$ for differentiation and isolation of pYV-bearing cells from pYV-less cells.

Day 3

(i) Using a stereomicroscope, red pinpoint colonies were examined to ensure Lcr and CR uptake. Using a sterile loop, 2-3 red pinpoint colonies were then inoculated into sterile $10 \mathrm{~mL}$ of $\mathrm{BHI}$ broth.

(ii) The broth was inoculated and incubated at $28^{\circ} \mathrm{C}$ for $18-24 \mathrm{~h}$.

Day 4

(i) The overnight culture was divided into three portions: frozen stock cultures, working stock cultures, and cells used for PCR assay and for expression of pYV-encoded virulent phenotypic characteristics including Lcr, CR uptake, and CV binding.

(ii) Frozen stock cultures: $5 \mathrm{~mL}$ of overnight culture was mixed with equal portions of BHI broth and 20\% glycerol and dispensed into $500 \mu \mathrm{L}$ portions for storage at $-80^{\circ} \mathrm{C}$.

(iii) Working stock cultures: using a $10 \mu \mathrm{L}$ loop, cells were streaked on CRMOX and CR-BHO. The plates were incubated for $48 \mathrm{~h}$ at $37^{\circ} \mathrm{C}$. Plates were then stored at $2^{\circ} \mathrm{C}$ for future use. Plates can be stored for 15 days for CR-MOX and 30 days for CR-BHO.

(iv) PCR assay: $1 \mathrm{~mL}$ portion of cells was centrifuged, and DNA was prepared for PCR assay. Presence of pYV was confirmed by PCR assay targeting the virF gene in pYV.

(v) The presence of pYV was also confirmed by demonstrating expression of phenotypic virulence characteristics including colonial morphology, CV binding, Lcr, and CR binding.

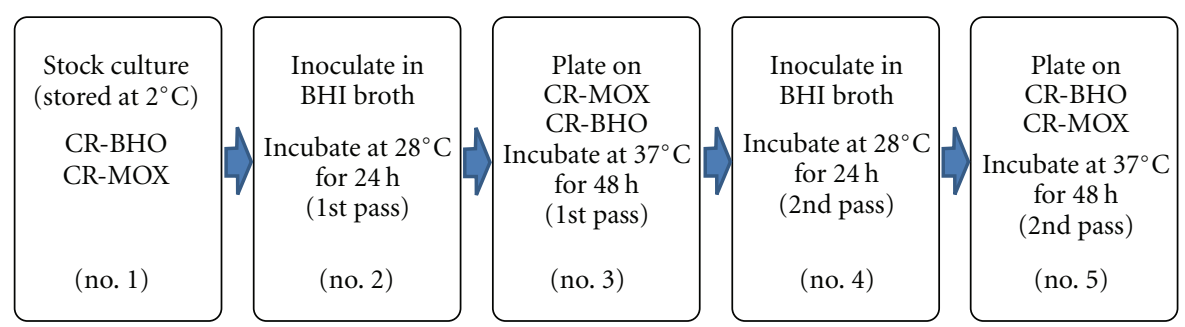

FIgUre 5: Confirmation of pYV in Y. pestis, [30].

after each passage was monitored and confirmed at every step of culture transfer (no. 2-5) by the PCR assay for pYV and by the expression of pYV-associated phenotypic virulence characteristics, including Lcr, CR uptake, and CV binding. The PCR data for the presence of PYV is shown in Figure 1 (lanes 7-10). PCR results confirm that primers amplified a 591-base pair (bp) product from pYV (virF gene) for each phase of the culture as described above, and all PCR-positive clones on CR-MOX and CR-BHO showed their virulent phenotypic characteristics including Lcr, CR-uptake, and CV binding. The presence of the virF gene demonstrates the presence of pYV, which confers PYVassociated phenotypes.

In conclusion, the described procedure provides a method to ensure the selection of pYV-bearing strains of $Y$. pestis and for studying pYV-bearing $Y$. pestis without losing pYV during experimental procedures [30]. Although CR$\mathrm{BHO}$ is a better medium for subculturing pYV-bearing $Y$. pestis, the pYV-bearing red pinpoint colonies are more easily detectable on CR-MOX due to more intense absorption of $\mathrm{CR}$ in the cells [7]. Hence, the use of CR-MOX for the preparation of stock cultures and to monitor the selection of pYV is recommended for investigation on the growth of
pYV-bearing Y. pestis in food. Thus, this procedure will allow only the Lcr-CR-positive pYV-bearing clones to be used to study growth behavior, growth models, and related studies in food.

\section{Application of CR-MOX for Isolation/Detection of Yersinia pestis in Food}

Yersinia pestis can cause oropharyngeal plague as a result of the consumption or handling of meat from infected animals [2-5]. Thus, food intentionally contaminated by $Y$. pestis could have a significant role in the dissemination of human plague. Existing microbiological media designed for the selective isolation/detection of $Y$. pestis in food based on phenotypic analysis were found to be unsatisfactory. The purpose of this section is to review the development of alternative methods for identification/isolation of pYVbearing $Y$. pestis based on the ability of $Y$. pestis to bind CR on calcium-depleted CR-MOX under specific conditions.

At present, the World Health Organization (WHO) [31] recommends the use of brain heart infusion (BHA) sheep blood agar and MacConkey agar for the isolation of $Y$. pestis. These growth media are suitable for sterile food; 
however, the isolation of $Y$. pestis from nonsterile foods is complicated by the presence of background flora competing for nutrients in the medium. Thus, the numerous colonies grown on these nonselective media require additional testing for the identification of the pathogen. MacConkey agar possesses a certain degree of selectivity; but the presence of CV and bile salt restricts the growth of $Y$. pestis [32]. $Y$. pestis strains exhibit slow or no growth in vitro on both cefsulodin-irgasan-novobiocin (CIN) agar and irgasannystatin agar [32] selective media when tested in our laboratory [7]. This may be due to the levels of selective substances used in this media. The colonies formed on selective media require further tests to identify them as $Y$. pestis. These tests are time consuming, costly, and labor intensive since a large number of presumptive colonies must be screened.

The calcium concentration in CR-BHO $\left(234 \mu \mathrm{M} \mathrm{Ca}^{2+}\right)$ is relatively low, whereas in CR-MOX, sodium oxalate is used to sequester the calcium, making the medium calcium deficient $[7,16]$. The comparison of CR uptake on calcium deficient CR-BHO and calcium-depleted CR-MOX among $Y$. pestis, $Y$. pseudotuberculosis, and $Y$. enterocolitica showed that this virulent phenotype is seen in pYV-positive strains of $Y$. pestis only when plated on calcium-depleted CR-MOX [7]. Thus, the CR uptake in Y. pestis is more dependent on calcium depletion than that of $Y$. enterocolitica and $Y$. pseudotuberculosis. Therefore, specific CR uptake on CR-MOX by $Y$. pestis can be used to differentiate $Y$. pestis from $Y$. enterocolitica and $Y$. pseudotuberculosis [7]. This would provide diagnostic value as follows: the suspected food samples are plated on CR-BHO and CR-MOX. If the colonies show $\mathrm{CR}$ uptake only on $\mathrm{CR}-\mathrm{MOX}$ at $37^{\circ} \mathrm{C}$ after $48 \mathrm{~h}$ of cultivation, then those $\mathrm{CR}^{+}$colonies can be isolated and identified as $Y$. pestis strains [7]. This technique will enhance the isolation/detection of $Y$. pestis strains in the presence of competing microflora by the proper selection of media and incubation times. The $\mathrm{CR}^{+} Y$. pestis clones can be further confirmed by PCR targeting the $Y$. pestis specific plasmid-encoded plasminogen activator gene [33]. To show the specificity of CR uptake by $Y$. pestis on CR-MOX, several species of bacteria including a number of foodborne pathogens were tested. These nonYersinia species did not form red pinpoint colonies and did not form a white border around the red center of the colony on CR-MOX [7, 17]. Furthermore, this method of isolation/detection for $Y$. pestis in food was verified by recovering the organism from artificially contaminated sterilized ground beef [29]. Thus, CR uptake on CRMOX by $Y$. pestis provides a microbiological method for the isolation/detection of this pathogen. In conclusion, the specific CR uptake of $Y$. pestis in a calcium-deficient medium provides a screening medium to isolate, detect, and differentiate this pathogen from $Y$. enterocolitica and $Y$. pseudotuberculosis, and this method is also applicable to food.

\section{Disclosure}

Mention of trade names or commercial products in this publication is solely for the purpose of providing specific information and does not imply recommendation or endorsement by the US Department of Agriculture.

\section{References}

[1] B. Wren, "The yersiniae-a model genus to study the rapid evolution of bacterial pathogens," Nature Reviews Microbiology, vol. 1, no. 1, pp. 55-64, 2003.

[2] A. B. Christie, T. H. Chen, and S. S. Elberg, "Plague in camels and goats: their role in human epidemics," Journal of Infectious Diseases, vol. 141, no. 6, pp. 724-726, 1980.

[3] A. Arbaji, S. Kharabsheh, S. Al-Azab et al., "A 12-case outbreak of pharyngeal plague following the consumption of camel meat, in north-eastern Jordan," Annals of Tropical Medicine and Parasitology, vol. 99, no. 8, pp. 789-793, 2005.

[4] A. A. B. Bin Saeed, N. A. Al-Hamdan, and R. E. Fontaine, "Plague from eating raw camel liver," Emerging Infectious Diseases, vol. 11, no. 9, pp. 1456-1457, 2005.

[5] T. Leslie, C. A. Whitehouse, S. Yingst et al., "Outbreak of gastroenteritis caused by Yersinia pestis in Afghanistan," Epidemiology and Infection, vol. 139, no. 5, pp. 1-8, 2011.

[6] M. Galimand, A. Guiyoule, G. Gerbaud et al., "Multidrug resistance in Yersinia pestis mediated by a transferable plasmid," New England Journal of Medicine, vol. 337, no. 10, pp. 677-680, 1997.

[7] S. Bhaduri and C. H. Sommers, "Detection of Yersinia pestis by comparison of virulence plasmid (pYV/PCD)-associated phenotypes in Yersinia species," Journal of Food Safety, vol. 28, no. 3, pp. 453-466, 2008.

[8] R. R. Brubaker, "Yersinia pestis and bubonic plague," in The Prokaryotes, M. Dworkin, S. Falkow, E. Rosenberg, and E. Stackebrandt, Eds., vol. 6, chapter 3.3.14, pp. 399-442, Springer, New York, NY, USA, 2006.

[9] S. W. Bearden and R. D. Perry, "Laboratory maintenance and characterization of Yersinia pestis," Current Protocols in Microbiology, no. 11, pp. 5B.1.1-5B.1.13, 2008.

[10] R. D. Perry and J. D. Fetherston, "Yersinia pestis-etiologic agent of plague," Clinical Microbiology Reviews, vol. 10, no. 1, pp. 35-66, 1997.

[11] R. M. Robins-Browne, "Yersinia enterocolitica," in Food Microbiology, Fundamentals and Frontiers, M. P. Doyle, L. R. Beuchat, and T. J. Montville, Eds., pp. 215-245, ASM Press, Washington, DC, USA, 2nd edition, 2011.

[12] E. Carniel, "Y. enterocolitica and Y. pseudotuberculosis Enteropathogenic yersiniae," in The Prokaryotes, M. Dworkin, S. Falkow, E. Rosenberg, and E. Stackebrandt, Eds., chapter 3.3.13, pp. 270-398, Springer, New York, NY, USA, 2006.

[13] S. Bhaduri, "Pathogenic Yersinia enterocolitica," in Guide to Foodborne Pathogens, R. G. Labbe and S. Garcia, Eds., pp. 245255, John Wiley and Sons, New York, NY, USA, 2001.

[14] M. Achtman, K. Zurth, G. Morelli, G. Torrea, A. Guiyoule, and E. Carniel, "Yersinia pestis, the cause of plague, is a recently emerged clone of Yersinia pseudotuberculosis," Proceedings of the National Academy of Sciences of the United States of America, vol. 96, no. 24, pp. 14043-14048, 1999.

[15] S. Bhaduri, "A comparison of sample preparation methods for PCR detection of pathogenic Yersinia enterocolitica from ground pork using swabbing and slurry homogenate 
techniques," Molecular and Cellular Probes, vol. 17, no. 2-3, pp. 99-105, 2003.

[16] S. Bhaduri, C. Turner-Jones, M. M. Taylor, and R. V. Lachica, "Simple assay of calcium dependency for virulent plasmidbearing clones of Yersinia enterocolitica," Journal of Clinical Microbiology, vol. 28, no. 4, pp. 798-800, 1990.

[17] S. Bhaduri, C. Turner-Jones, and R. V. Lachica, "Convenient agarose medium for simultaneous determination of the lowcalcium response and congo red binding by virulent strains of Yersinia enterocolitica," Journal of Clinical Microbiology, vol. 29, no. 10, pp. 2341-2344, 1991.

[18] S. Bhaduri, B. Cottrell, and A. R. Pickard, "Use of a single procedure for selective enrichment, isolation, and identification of plasmid-bearing virulent Yersinia enterocolitica of various serotypes from pork samples," Applied and Environmental Microbiology, vol. 63, no. 5, pp. 1657-1660, 1997.

[19] S. Bhaduri and B. Cottrell, "Direct detection and isolation of plasmid-bearing virulent serotypes of Yersinia enterocolitica from various foods," Applied and Environmental Microbiology, vol. 63, no. 12, pp. 4952-4955, 1997.

[20] S. Bhaduri, L. K. Conway, and R. V. Lachica, "Assay of crystal violet binding for rapid identification of virulent plasmidbearing clones of Yersinia enterocolitica," Journal of Clinical Microbiology, vol. 25, no. 6, pp. 1039-1042, 1987.

[21] T. Nesbakken, G. Kapperud, H. Sorum, and K. Dommarsnes, "Structural variability of 40-50 Mdal virulence plasmids from Yersinia enterocolitica. Geographical and ecological distribution of plasmid variants," Acta Pathologica Microbiologica et Immunologica Scandinavica, vol. 95, no. 3, pp. 167-173, 1987.

[22] M. Fredriksson-Ahomaa and H. Korkeala, "Low occurrence of pathogenic Yersinia enterocolitica in clinical, food, and environmental samples: a methodological problem," Clinical Microbiology Reviews, vol. 16, no. 2, pp. 220-229, 2003.

[23] K. Juríková, B. Gottwaldová, S. Jacková, and J. Šubík, "Characterization of Yersinia enterocolitica isolated from the oral cavity of swines in Slovakia," International Journal of Food Microbiology, vol. 24, no. 3, pp. 419-424, 1995.

[24] E. Koeppel, R. Meyer, J. Luethy, and U. Candrian, "Recognition of pathogenic Yersinia enterocolitica by crystal violet binding and polymerase chain reaction," Letters in Applied Microbiology, vol. 17, no. 5, pp. 231-234, 1993.

[25] S. D. Weagant, P. Feng, and J. T. Stanfield, Yersinia enterocolitica, and Yersinia pseudotuberculosis in Bacteriological Manual, Revision A. Food and Drug Administration, AOAC International, Gathersburg, Md, USA, 8th edition, 1998.

[26] J. J. P. Kwaga and J. O. Iversen, "Laboratory investigation of virulence among strains of Yersinia enterocolitica and related species isolated from pigs and pork products," Canadian Journal of Microbiology, vol. 38, no. 2, pp. 92-97, 1992.

[27] S. C. Straley, "The low- $\mathrm{Ca}^{2+}$ response virulence regulon of human-pathogenic yersiniae," Microbial Pathogenesis, vol. 10, no. 2, pp. 87-91, 1991.

[28] S. C. Straley, E. Skrzypek, G. V. Plano, and J. B. Bliska, "Yops of Yersinia spp. pathogenic for humans," Infection and Immunity, vol. 61, no. 8, pp. 3105-3110, 1993.

[29] S. Bhaduri, "Effect of fat in ground beef on the growth and virulence plasmid (pYV) stability in Yersinia pestis," International Journal of Food Microbiology, vol. 136, no. 3, pp. 372-375, 2010.

[30] S. Bhaduri, K. Chaney-Pope, and J. L. Smith, "A procedure for monitoring the presence of the virulence plasmid ( $\mathrm{pYV}$ ) in
Yersinia pestis under culture conditions," Foodborne Pathogens and Disease, vol. 8, no. 3, pp. 459-463, 2011.

[31] D. T. Dennis, K. L. Gage, N. Gratz, J. D. Polan, and E. Tikhomriov, Plague Manual: Epidemiology, Distribution, Surveillanceand Control, World Health Organization, Geneva, Switzerland, 1999.

[32] R. Ber, E. Mamroud, M. Aftalion et al., "Development of an improved selective agar medium for isolation of Yersinia pestis," Applied and Environmental Microbiology, vol. 69, no. 10, pp. 5787-5792, 2003.

[33] C. Loiez, S. Herwegh, F. Wallet, S. Armand, F. Guinet, and R. J. Courcol, "Detection of Yersinia pestis in sputum by real-time PCR," Journal of Clinical Microbiology, vol. 41, no. 10, pp. 4873-4875, 2003. 


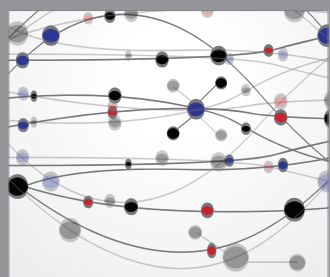

The Scientific World Journal
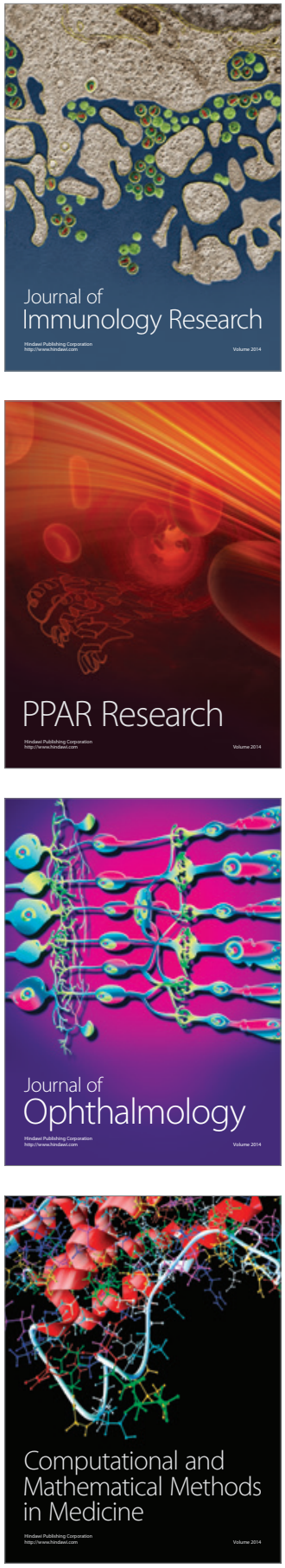

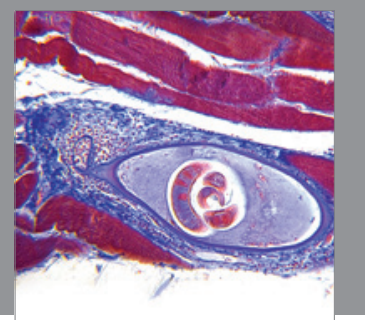

Gastroenterology

Research and Practice
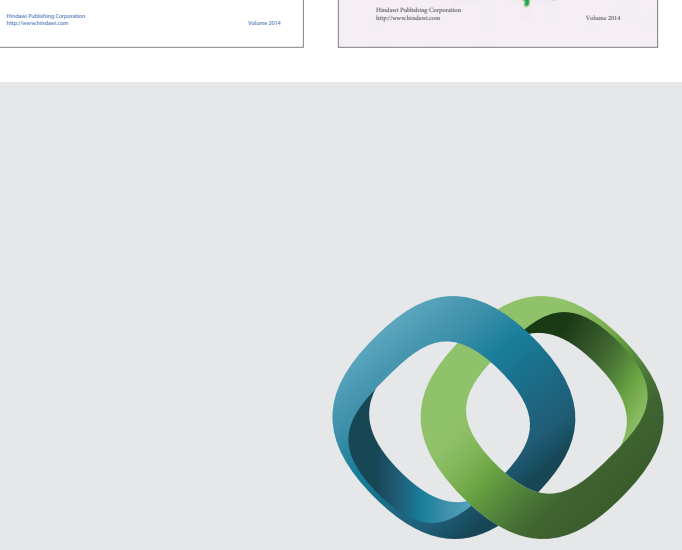

\section{Hindawi}

Submit your manuscripts at

http://www.hindawi.com
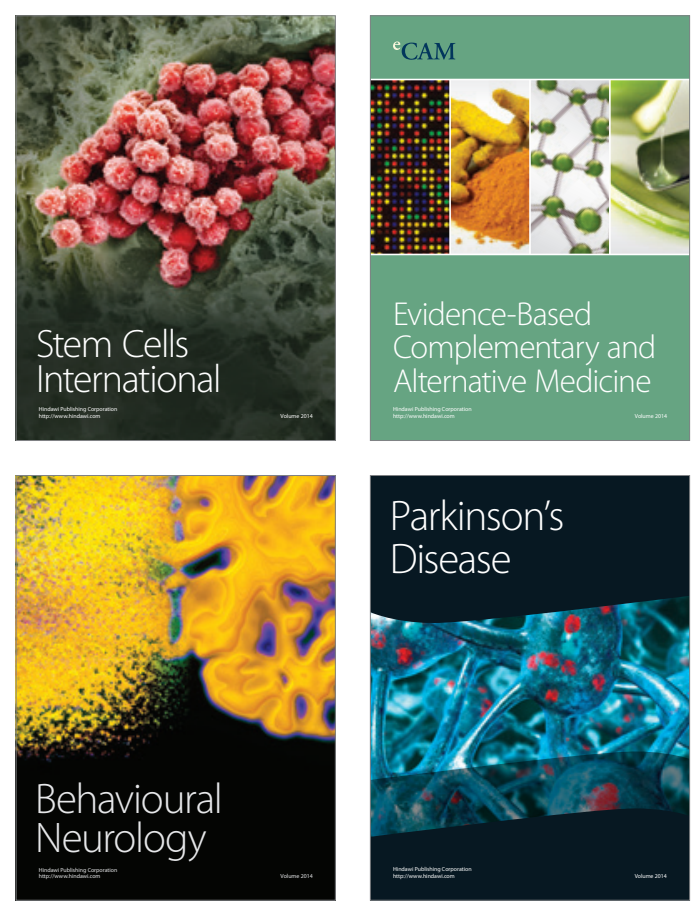

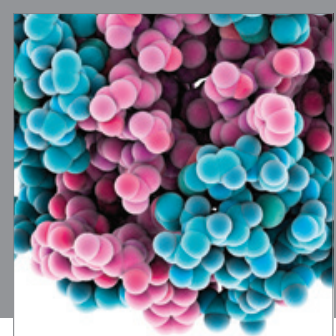

Journal of
Diabetes Research

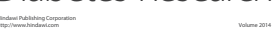

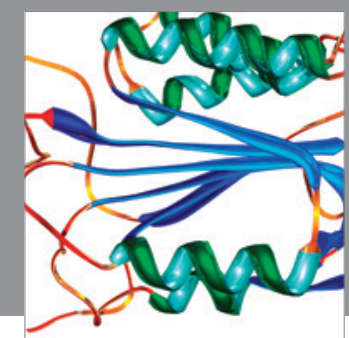

Disease Markers
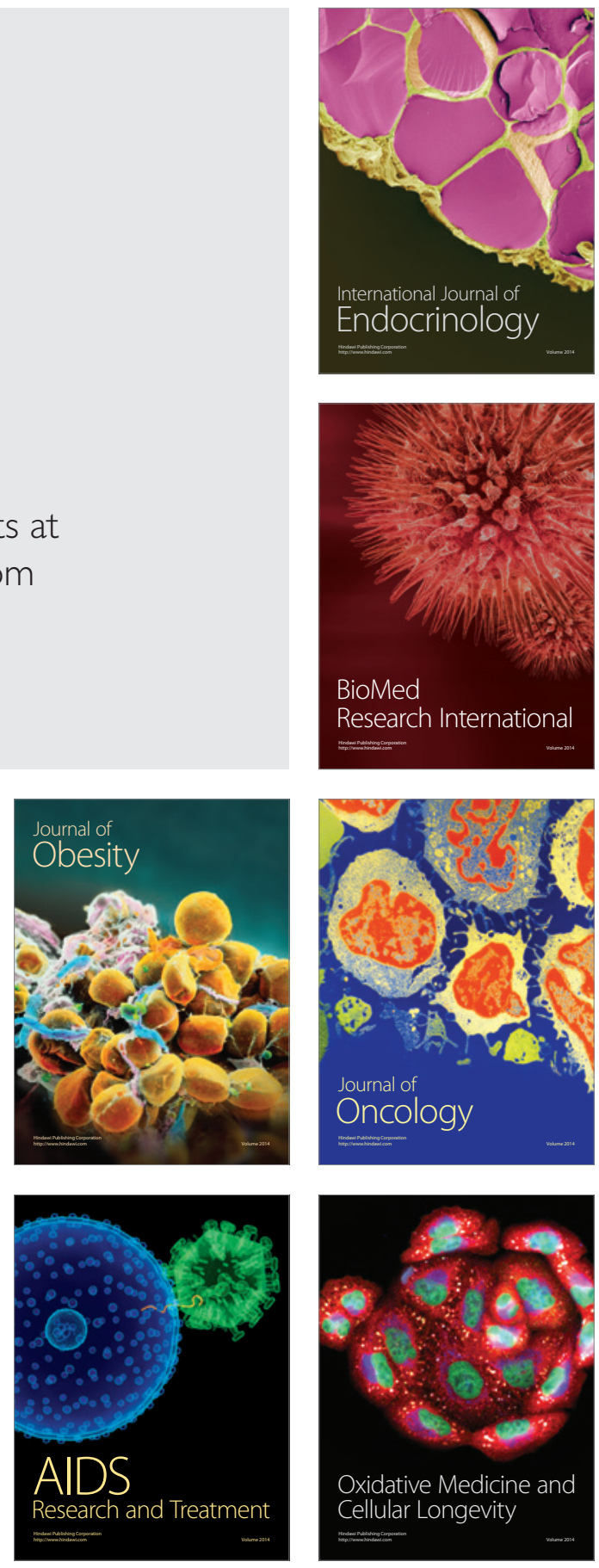